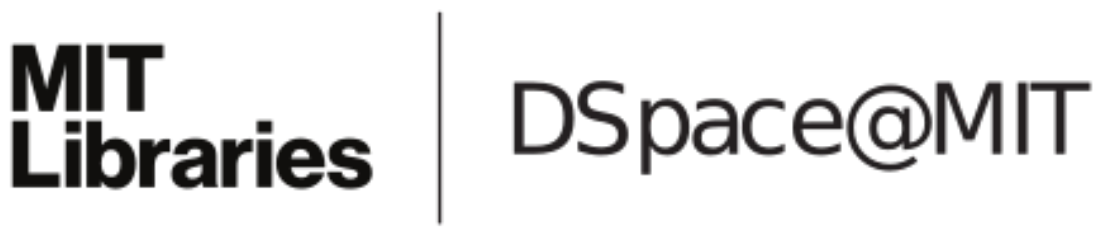

\author{
MIT Open Access Articles
}

Climate-Conscious Urban Growth Mitigates Urban Warming: Evidence from Shenzhen, China

The MIT Faculty has made this article openly available. Please share how this access benefits you. Your story matters.

Citation: Zhou, Yulun, Huang, Bo, Wang, Jionghua, Chen, Bin, Kong, Hui et al. 2019. "ClimateConscious Urban Growth Mitigates Urban Warming: Evidence from Shenzhen, China." Environmental Science and Technology, 53 (20).

As Published: http://dx.doi.org/10.1021/acs.est.9b01645

Publisher: American Chemical Society (ACS)

Persistent URL: https://hdl.handle.net/1721.1/133019

Version: Author's final manuscript: final author's manuscript post peer review, without publisher's formatting or copy editing

Terms of use: Creative Commons Attribution-Noncommercial-Share Alike 


\title{
Climate-Conscious Urban Growth Mitigates Urban Warming: Evidence from Shenzhen, China
}

\author{
A Preprint - Environmental Science \& Technology \\ [ Subject to Changes in the final version ] \\ Please Cite: http://dx.doi.org/10.1021/acs.est.9b01645
}

\author{
Yulun Zhou ${ }^{1 \dagger}$, Bo Huang ${ }^{12^{\dagger *}}$, Jionghua Wang ${ }^{23}$, Bin Chen ${ }^{4}$, Hui Kong ${ }^{5}$, Leslie Norford ${ }^{6}$ \\ ${ }^{1}$ Department of Geography \& Resource Management, The Chinese University of Hong Kong, Hong Kong, China \\ ${ }^{2}$ Institute of Space and Earth Information Science, The Chinese University of Hong Kong, Hong Kong, China \\ ${ }^{3}$ Faculty of Geosciences and Environmental Engineering, Southwest Jiaotong University, Chendu 611756, China \\ ${ }^{4}$ Department of Land, Air and Water Resources, University of California, Davis, CA 95616, USA \\ ${ }^{5}$ Singapore-MIT Alliance for Research and Technology (SMART), Singapore 138602 \\ ${ }^{6}$ Department of Architecture, Massachusetts Institute of Technology, Cambridge, MA 02139, USA \\ ${ }^{\dagger}$ Yulun Zhou and Bo Huang contributed equally to this manuscript and should be considered co-first authors. \\ * Corresponding author: Bo Huang: bohuang@cuhk.edu.hk
}

\begin{abstract}
Urban growth comes with significant warming impacts and related increases in air pollution concentrations, so many cities have implemented growth management to minimize 'sprawl' and its environmental consequences. However, controlling the amount of growth is costly. Therefore, in this paper, we focus on urban warming and investigate whether climate-conscious urban growth planning (CUGP), i.e., urban growth with the same magnitude but optimized spatial arrangements, brings significant mitigation effects. First, the classical spatial multi-objective land-use optimization (SMOLA) model is improved by integrating the spatially-, diurnally-, and compositionally-varying associations between land-use and their warming impacts. Then, we solve the improved model using the non-dominated genetic algorithm (NSGA-II) to generate urban growth plans with minimal warming impacts and minimal cost of change without reducing the amount of urban growth. Results show that climate-conscious urban growth brings $33.3 \pm 4.6 \%$ less warming impacts compared to unplanned urban growth in Shenzhen, China, and suggest a compact and spatially equalized development pattern. This study provides evidence that spatial planning tools such as the CUGP can help mitigate human impacts on the environment. Meanwhile, the improved SMOLA model could be applied to balance urban development and other environmental consequences such as air pollution.
\end{abstract}

\section{Acknowledgement}

Appreciation goes to Qianhui Liang (MIT) and Michael Seidenkühnel for engaging in artistic designs of the cover art. This study was supported by National Key R\&D Program of China (2017YFB0503605), which is gratefully acknowledged. 


\section{INTRODUCTION}

One of the most direct environmental consequences arising from massive global urbanization is urban warming, which leads to increased cooling energy demand, increased air pollution, and elevated public health risks associated with human exposure to high ambient temperatures. Urban heat island (UHI), the long-recognized effect that cities have higher air temperatures than their surrounding countrysides, is an extreme case of how land-use changes modify regional climate ${ }^{1}$. Reported in many global cities, exacerbated UHIs can be as dramatic as $10^{\circ} \mathrm{C}$ depending on the local weather conditions ${ }^{2,3}$. Such intense heat stress brings substantial health risks to urban dwellers, particularly during excessive heat events (EHEs), i.e., heat waves ${ }^{4}$. Exposure to extreme heat leaves the elderly, in particular, at risk of suffering heat cramps, heat exhaustion, and heatstroke. Temperature is also found to exert the strongest and most stable influence on $\mathrm{PM}_{2.5}$ concentrations in all seasons amongst meteorological factors ${ }^{5}$. Researchers have long sought to mitigate urban warming, and many studies have suggested such promising mitigation measures as green roof, reflective streets, and increased green space with optimized positioning ${ }^{6-12}$. However, existing studies on mitigating urban warming paid insufficient attention to making low-cost improvements to future urban growth plans. Also, many studies have not considered the extreme case of EHEs when heat-related deaths are most likely to occur ${ }^{13}$.

Urban growth - the expansion of a metropolitan or suburban area into the surrounding environment - is an economic phenomenon inextricably linked with the process of urbanization ${ }^{14}$. Urban lands - developed, built-up areas with a density of human structures such as houses, buildings, and roads - are primary heat sources in cities ${ }^{15}$. Urban growth increases urban temperatures significantly by reducing green space, altering surface albedo and geometry compared to rural surfaces ${ }^{15}$. The associations between urban land-uses and their warming effects have been studied extensively ${ }^{16-18}$. Urban growth is found to be consistently associated with a substantial temperature increase ${ }^{11,19,20}$, and in newly urbanized areas of the city, such an increase can be comparable to the increase under the highest greenhouse gas emissions scenario (RCP8.5) ${ }^{21}$. Such temperature increases vary with time of day ${ }^{16}$ and land-use compositions ${ }^{17,22}$, i.e., the abundance and variety of land cover features. The development density of urban lands is a critical compositional factor in the variability of urban temperatures; higher temperatures often occur in dense urban areas, where the population density is also higher. However, nearly half of the studies on urban warming impacts of urbanization have ignored the variability in urban development density ${ }^{23}$, which may underestimate the magnitude of the warming impacts of urban lands, especially in the densest areas. Besides the composition, the spatial configuration ${ }^{17,24,25}$ of urban lands, that is, their spatial arrangement and distribution, can affect the magnitude of urban warming. For example, comparison results from regional climate simulations indicate that a compact mode of urban growth has significant potential in moderating the mean-areal urban warming ${ }^{26,27}$. Empirical data analyses also show that sprawling cities are more vulnerable to heat stress during EHEs compared to their compact counterparts ${ }^{4}$. 
Future urban growth with careful spatial arrangements may mitigate its warming impacts without having to reduce the total amount of the growth, yet we know little about the optimal effectiveness and efficiency of this approach. Recent developments in spatial optimization methods have made it possible to formulate the land-use planning problem as a spatial multi-objective land-use allocation (SMOLA) problem guided by a selected set of objectives about sustainability.

Land-use optimization problems were first articulated in the 1960s when the linear programming (LP) model of land-use design was proposed ${ }^{28}$. Later developments in spatial optimization methods have introduced the location-allocation model $^{29}$ and their generalization to multi-objective optimizations in which the trade-offs among multiple objectives were considered ${ }^{30-34}$. In early practices of solving multi-objective problems using LP models $^{35}$, it was a major issue to quantify the relative weights of the selected objectives. Then, SMOLA models based on Pareto optimality were proposed and became popular for land-use problems $s^{11,34,36-39}$, since the objectives were independent of each other, no weights were needed, and a series of equally good solutions, called "the Pareto Front", can be generated simultaneously. Since the environmental impacts of urbanization are increasingly drawing public attention, there has been growing interest in the use of SMOLA models to mitigate negative impacts. For instance, Zhang and Huang ${ }^{11}$ first applied a spatial multi-objective optimization model to mitigate urban heat island effects. Zhang et al. ${ }^{12}$ demonstrated that increased greenness with optimized distributions could be a useful urban warming mitigation measure. However, most existing studies ignored the heterogeneity in the objective functions, i.e., the empirical models used to assess the environmental impacts of optimized plans, except for Zhang et al. ${ }^{12}$, who included different assessment functions for daytime and nighttime temperatures and enabled identification of trade-off solutions that balance diurnal cooling benefits. Therefore, the assumption that these objective functions are fixed is not always valid, especially when dealing with environmental objectives, since such environmental responses as urban warming could contain substantial spatial, diurnal and compositional variances. Not considering such heterogeneous associations in environment-related spatial optimization problems may lead to two main problems: 1) the fixed functions may not provide accurate environmental impact assessments of the optimized plans, and so the SMOLA model may fail to generate plans with real improvements. 2) the solution space is limited by the fixed functions, leading to limited improvements in environmental objectives.

Therefore, this paper has two objectives. First, we investigate the spatially-, diurnally-, and compositionally-varying temperature-land-use relationships and improve the classical SMOLA model by integrating such varying relationships. Second, using the improved SMOLA model, we systematically examine the effectiveness and cost of the so-called climate-conscious urban growth planning (CUGP) as a possible mitigation measure for urban warming. This investigation is significant and timely for two main reasons: 
1) If planned wisely, the expected urban growth in the next two decades will provide a significant opportunity for urban warming mitigation.

2) It is now possible to tap the potential climate benefits from spatial urban growth planning with extensive results on temperature-land-use relationships and recent developments in spatial optimization.

The remainder of this paper is organized as follows. Section 2 introduces the materials and methods used in the investigation. Sections 3 and 4 present results and discussions on climate-conscious urban growth based on our case study in Shenzhen, China. Section 5 present the conclusions.

\section{MATERIAL AND METHODS}

The study was conducted in two steps. First, we modeled the temperature-land-use relationship to allow the warming effects of urban growth to be estimated empirically. Then, we measured the effectiveness of the CUGP for urban warming mitigation by estimating the warming impacts of urban growth with (Experimental Scenario) and without (Baseline Scenario) optimized spatial arrangements and comparing them with each other (Figure S1).

\section{Notations}

$136 \quad i \quad i \in\{1,2, \ldots, N\}$; cell locations, where $N$ is the total number of cells in the study area.

$137 L_{i} \quad$ land-use at location $i$ in the status quo.

$138 L_{i}^{\prime} \quad$ land-use at location $i$ in the optimized plan.

$139 \quad I_{i} \quad$ land-use intensity index at location $i$ in the status quo.

$140 \quad I_{i}^{\prime} \quad$ land-use intensity index at location $i$ in the optimized plan.

$141 \quad e_{i} \quad$ elevation at location $i$.

$142 s_{i} \quad$ slope at location $i$.

$143 \quad P_{l m} \quad$ the probability that land-use $l$ is an immediate neighbor of land-use $m$ in the status quo.

$144 \quad P_{l m}^{\prime} \quad$ the probability that land-use $l$ is an immediate neighbor of land-use $m$ in the optimized plan.

$145 \mathbf{U} \quad$ set of urban lands, including high-, mid-, and low-density urban lands.

1472.1 Site description. Shenzhen (east longitude: $113^{\circ} 46^{\prime}$ to $114^{\circ} 37^{\prime}$, north latitude: $22^{\circ} 27^{\prime}$ to $22^{\circ} 52^{\prime}$ ) 148 is a fast-developing post-reform city in south China (Figure 1). As a sub-tropical city with a warm, 149 monsoon-influenced, humid climate, Shenzhen has an average annual temperature of $23^{\circ} \mathrm{C}$, and the 150 local summer lasts as long as six months. Luo and $\mathrm{Lau}^{38}$ observed a significant increase in 151 frequency, duration, and intensity of heatwaves in Southern China in the past 40 years; over 50\% 152 of the increase was contributed by urbanization. Within territory of $2050 \mathrm{~km}^{2}$, the population in 153 Shenzhen has exploded from 2.39 million in 1995 to 10.22 million in 2010 and is projected to reach $154 \quad 12.67$ million by 2030 . 


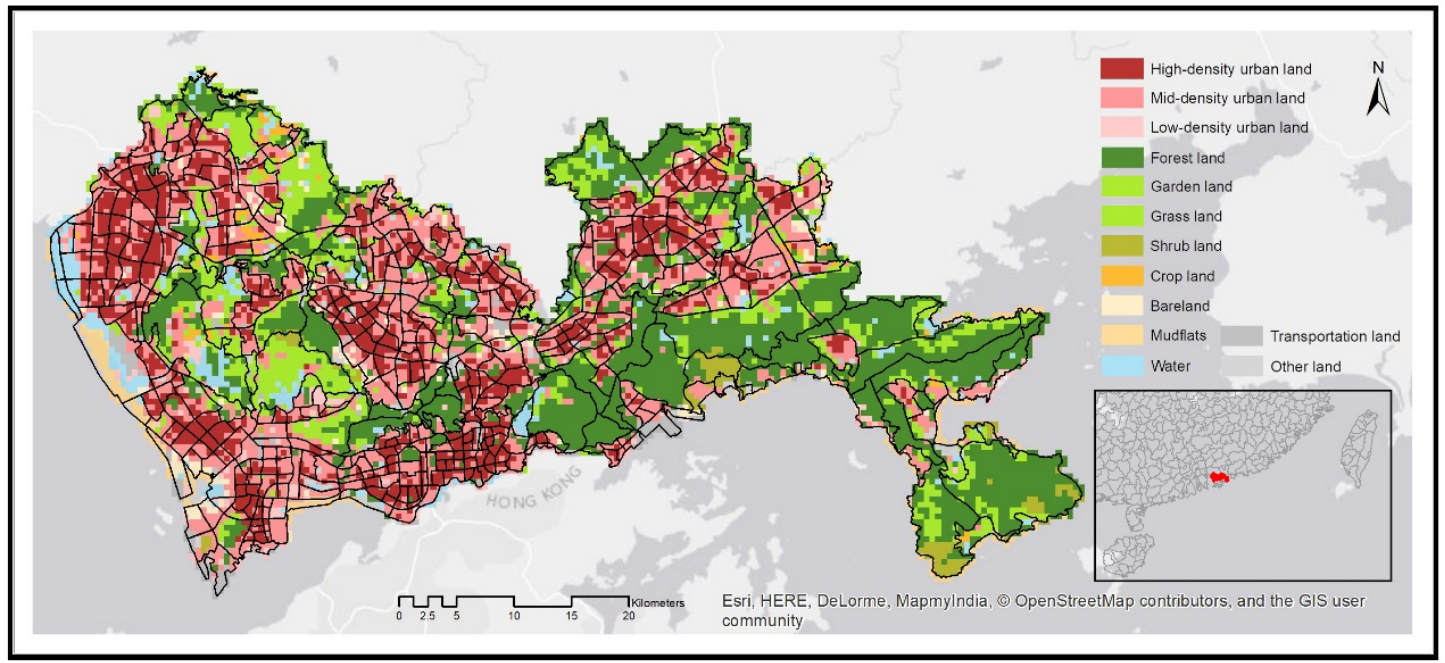

Figure 1 Land-use map of Shenzhen, a sub-tropical city in south China, in 2010. The used urban subset represents sub-districts in Shenzhen ${ }^{44}$.

2.2 Data. We focused on the strongest EHE identified in the year of 2010, which lasted 15 days from June $30^{\text {th }}$ to July $15^{\text {th }}$. The EHE was identified by Luo and Lau ${ }^{38}$ using observations from 86 national ground monitoring stations in Guangdong Province over a 40-year period from 1970 to 2010. Satellite-retrieved LST maps and land-use maps were mainly used for the empirical modeling of temperature-land-use relationships. The Land Surface Temperature (LST) was obtained from EOS-Aqua-MODIS C6 composite products (MYD11A2) with a spatial resolution of 30 arc-second $(\sim 1 \mathrm{~km})$. The LST map was retrieved from clear-sky (99\% confidence) observations at 1:30 (nighttime) and 13:30 (daytime) local solar time by using a refined generalized split-window algorithm ${ }^{41}$. We resampled the spatial resolution of LST maps to $500 \mathrm{~m}$ to overlay with the landuse map. Besides, the urban land-use map was retrieved from the official land-use map of Shenzhen in 2010, which is a product of field survey and systematic quality control. We reclassified the urban land category into three subcategories based on their varying development density (please refer to Section S1.2 for more details). The spatial resolution of the land-use map is $500 \mathrm{~m}$. A digital elevation map (DEM) of Shenzhen in 2010 with the spatial resolution of $30 \mathrm{~m}$ was utilized to quantify terrain limits for urban developments. The land-use map and the DEM data are all products of field surveys with systematic quality assurance and control.

2.3 The temperature-land-use relationship. Since the assumption of the existence of a spatially fixed relationship is not always true, especially when dealing with geographical data and such phenomena as urban warming, we applied both global and local modeling methods to estimate the temperature-land-use relationship to find the better-performing model. Urban temperatures can be characterized using air temperature or $\mathrm{LST}^{15,19}$. We used LST as the indicator of urban warming because it is more directly linked to the land-use changes induced by urban growth via the alteration of the physical and biophysical processes ${ }^{19}$. 
We took LST as the response variable and calculated 13 land-use indices (Table S1) as predictor variables using the Inversed-Distance-Weighted (IDW) sum to consider not only the local land-use but also its immediate neighbors. We first applied stepwise ordinary least squared regression (OLS) (Eq. 1), one of the most commonly used global modeling approaches, to estimate the temperatureland-use relationship, assuming the existence of a spatially fixed relationship:

$L S T_{i}=\sum_{l} \gamma_{l} I_{l, i} \quad$ (Eq. 1)

where $i$ indicates spatial locations within the study area and $I_{l, i}$ is the land-use intensity for the $l^{\text {th }}$ landuse type and $\gamma_{l}$ is its spatially fixed coefficient. $I_{l, i}$ is the land-use intensity for the $l^{\text {th }}$ land-use type and $\gamma_{l}$ is its spatially fixed coefficient. The estimated temperature-land-use relationship was selfvalidated using 10 -fold cross-validation (CV). A Python tool was programed for the stepwise OLS estimations equipped with 10 -fold $\mathrm{CV}$.

Then, we applied semi-parametric geographically weighted regression (sGWR) ${ }^{42}$ as the local modeling approach to estimate the temperature-land-use relationship. GWR expands the traditional cross-sectional regression model (Eq. 1) to allow for local variations in the estimated parameters and is found to be a more appropriate analytical framework in conducting research involving multiple spatial data with autocorrelated structures ${ }^{16}$. Unlike regular GWR, sGWR allows the simultaneous fitting of mixed spatially varying and fixed coefficients in the same model (Eq. 2), as follows,

where $I_{k, i}$ and $\beta_{k}$ are the $k^{\text {th }}$ local explanatory variable and its coefficient. The coefficients vary depending on the geographical location, $\left(u_{i}, v_{i}\right)$. The GWR estimations were implemented using the GWR4 package (version 4.0.90, http://gwr.maynoothuniversity.ie/gwr4-software/).

2.4 The SMOLA model improved for environmental objectives. The SMOLA model was improved for considering urban warming or other related environmental objectives by capturing and integrating the spatially-, diurnally-, and compositionally varying environmental responses to land-use changes. In doing so, urban growth plans were optimized based on more reliable environmental impact assessments; the solution space was also enlarged with more combinatorial possibilities. The integration of varying environmental responses into the improved SMOLA model may lead to very different optimization results and larger objective improvements compared to the classical SMOLA model. We solved the SMOLA model using the non-dominated sorting genetic algorithm (NSGA-II), introduced for this purpose by Cao et al. ${ }^{34}$. Unlike classical multi-objective optimization methods, the NSGA-II algorithm generates a diverse population of equally optimal 
solutions instead of a single optimal solution, leaving human decision-makers to make the final decision. For details of the NSGA-II-based SMOLA model, please refer to the Supplementary Material.

2.5 Warming impacts of unplanned urban growth. In the baseline scenario, we first simulated urban land-use plans with $10 \%$ more unplanned urban land area. The new urban lands were iteratively added to the status quo of Shenzhen, 2010 (Figure 1), using a random boundary-based urban growth operator, where all new urban lands were restricted to the boundary area of existing urban lands. The unplanned urban growth was also subject to the three feasibility constraints in Section 2.6. Then, the new urban lands were randomly assigned as high-density, mid-density or low-density urban lands, while the average development density of the entire land-use plan was constrained to be at least equal to or higher than that of the status quo. The process was repeated to generate $N$ different plans with unplanned urban growth, where $N$ is the fine-tuned population size in each generation of the spatial optimization model explained in Section 2.4.

The warming impacts of unplanned urban growth were evaluated empirically using the temperatureland-use relationship estimated in the previous section.

2.6 Warming impacts of climate-conscious urban growth. In the experimental scenario, using an improved SMOLA model, the spatial arrangements of the unplanned urban growth simulated in the previous section were optimized under four objectives: to minimize daytime LST ( $\left.L S T_{d}\right)$, to minimize nighttime LST $\left(L S T_{n}\right)$, to maximize land-use compatibility, and to minimize changing cost. Both daytime and nighttime LST were minimized simultaneously to consider the diurnal temperature trade-off ${ }^{12}$. Besides the climate objectives, the number of land-use changes was also minimized to maximize the efficiency of optimized changes. In addition, the compatibility between adjacent land-uses was maximized, since there are land-use types that should not exist next to each other (e.g., industrial and residential). Rather than arbitrarily assigning the compatibility weights for each land-use pair $\left(P_{l m}\right)$, the weights were learned by summarizing their corresponding appearance probability in the status quo.

Three feasibility constraints proposed to limit the solution space of land-use plans:

1. The land demand of Shenzhen shall be satisfied. The increase of urban land area compared to the status quo should be equal to or larger than the required urban growth rate $\left(r_{g}\right)$ of $10 \%$. The average development density cannot be lower than that of the status quo.

2. Urban development cannot occur on rough terrain. Highlands, i.e., land units higher than $80 \mathrm{~m}$ in elevation, and hilly terrain, i.e., larger than 25 degrees in slope, were considered not suitable for urban developments. The $80 \mathrm{~m}$ was selected as the 95 percentiles of the elevation values of existing urban lands. The 25 degrees was set according to local 
government regulations.

3. Waterbody and croplands are preserved. No changes were allowed to existing water body due to their essential ecological benefits. Croplands are also preserved to secure urban food supply.

The objectives and constraints can be denoted as follows:

where $i$ indicates cell locations within the study area.

\section{RESULTS}

3.1 The estimated LST-land-use relationships. By considering the spatially varying effects, the spatially explicit models for both $L S T_{d}$ and $L S T_{n}$ fit the observations significantly better than their corresponding spatially fixed models and therefore provide more accurate predictions of LSTs (Section S2.1 for detailed results). The spatially explicit models provide good model fittings for both $L S T_{d}\left(\mathrm{R}^{2}=0.810\right)$ and $L S T_{n}\left(\mathrm{R}^{2}=0.725\right)$, while the spatially fixed model has a fair model fitting for $L S T_{d}\left(\mathrm{R}^{2}=0.537\right)$ and a poor model fitting for $L S T_{n}\left(\mathrm{R}^{2}=0.275\right)$. Since the spatially 
294 explicit models significantly outperform the spatially fixed models, they are selected as the objective

295 functions for assessing the warming impacts of urban land-use changes in the following SMOLA

296 model.

3.2 Cost-Effectiveness of the CUGP. Our evidence in Shenzhen, China shows that the CUGP can 299 be an effective urban climate mitigation measure. Both with $10 \%$ more urban lands, the unplanned 300 urban growth in the baseline scenario increased the average $L S T_{d}$ in Shenzhen by $0.21{ }^{\circ} \mathrm{C}$, while in 301 the experiment scenario, climate-conscious urban growth increased the average $L S T_{d}$ by $0.14^{\circ} \mathrm{C}$, which is $0.07 \pm 0.01^{\circ} \mathrm{C}(33.8 \pm 4.6 \%)$ less warming impacts. Results from the t-test showed that the $0.07^{\circ} \mathrm{C}$ difference was statistically significant $(\mathrm{p}<0.005)$.

The $0.07^{\circ} \mathrm{C}$ difference is not the cooling benefit on a single land unit; rather, it is the average cooling benefit averaged over all land units in Shenzhen. The cooling efficiency of each optimized land-use change is much more significant. The daytime $\overline{\Delta L S T}(E q .3)$ is $-1.24{ }^{\circ} \mathrm{C} /$ change, and the nighttime $\overline{\Delta L S T}$ is $-0.52^{\circ} \mathrm{C} /$ change. The CUGP is more effective during the daytime, since the $L S T_{d}$ is more variable and sensitive to land-use changes. Despite the diurnal trade-offs between $L S T_{d}$ and $L S T_{n}$, such decrease in $L S T_{d}$ was not achieved at the sacrifice of $L S T_{n}$, in fact, $L S T_{n}$ also decreased an average of $0.03^{\circ} \mathrm{C}$ after the optimization. Figure 2 maps daytime and nighttime LST changes caused by the CUGP. 

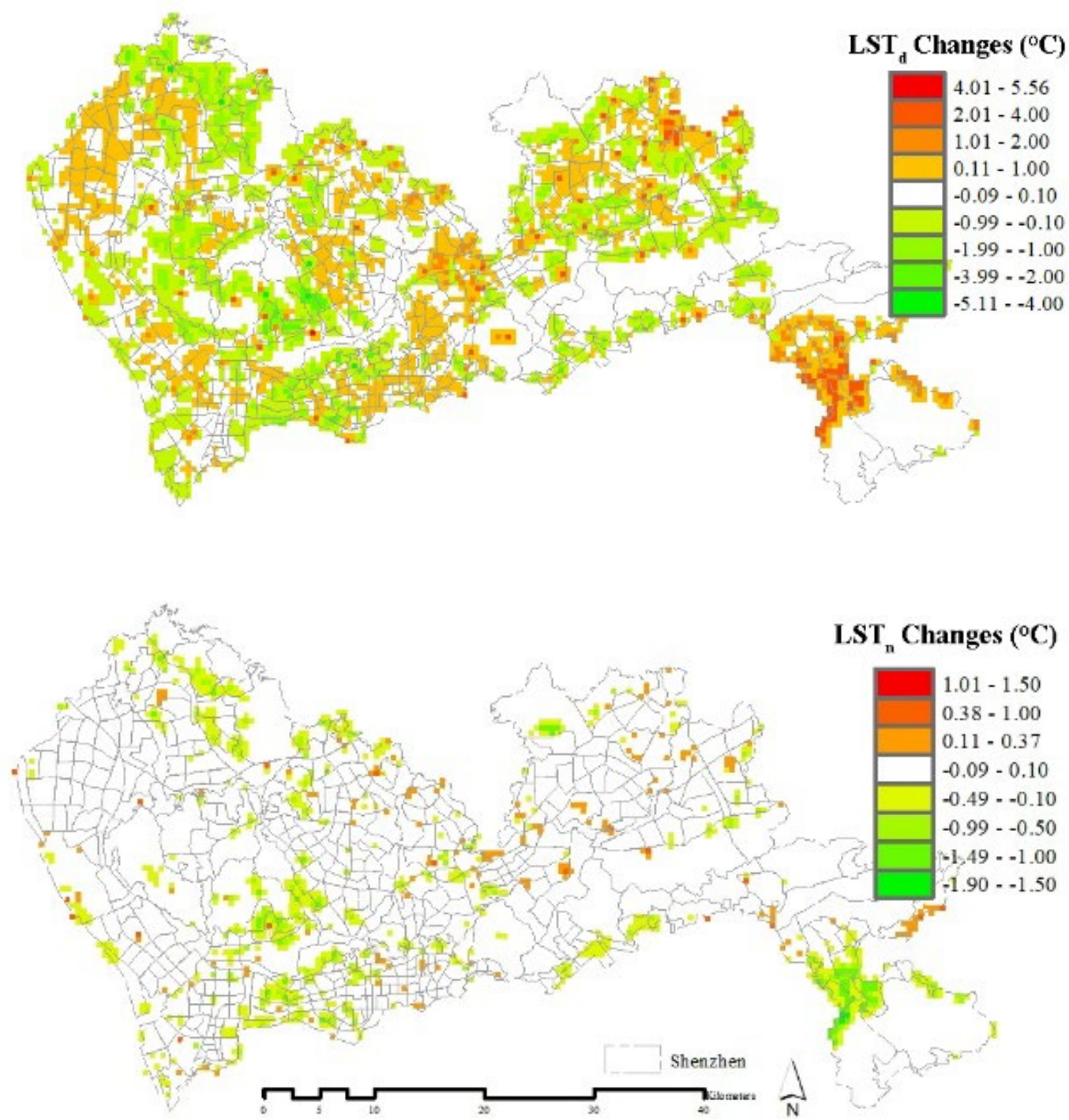

Figure 2 Changes in daytime LST $\left(L S T_{d}\right)$ and nighttime LST $\left(L S T_{n}\right)$ introduced by the CUGP, compared with warming impacts of unplanned urban growth.

318 In addition, we examined the cost-effectiveness of the CUGP (Figure 3). The effectiveness of the 319 CUGP grows non-linearly as the number of changes increases. We took the lowest average $L S T_{d}$ and $L S T_{\mathrm{n}}$ among all optimized urban growth plans as the optimal mitigation performance that can be achieved with the CUGP regardless of the number of land-use changes. To achieve $80 \%$ of the optimal effectiveness in both $L S T_{d}$ and $L S T_{\mathrm{n}}$ requires at least $56 \%$ of land-use changes. To achieve $50 \%$ of the optimal effectiveness in both $L S T_{d}$ and $L S T_{\mathrm{n}}$ requires at least $25 \%$ of land-use changes. 

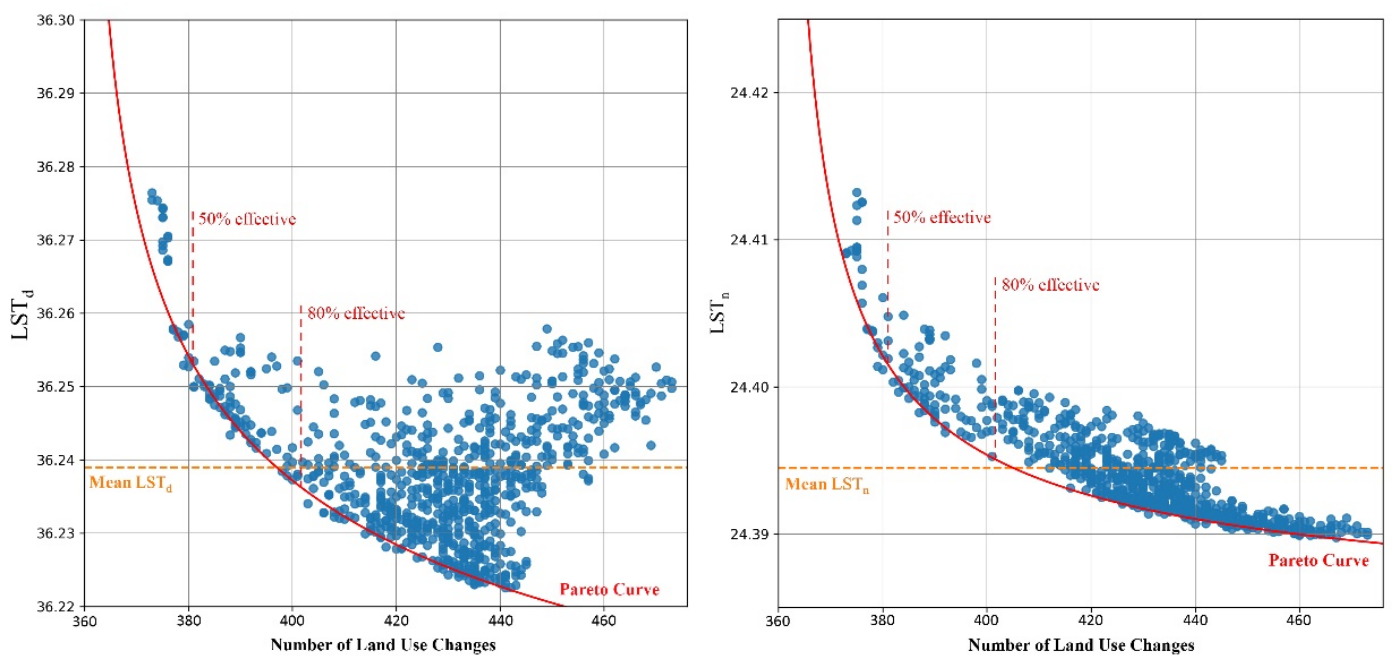

Figure 3 Cost-effectiveness of the CUGP for mitigating LST ${ }_{d}$ (left) and LST $T_{n}$ (right). Both two-dimentional Pareto fronts can be fitted almost perfectly with a Pareto curve $\left(R^{2}>0.95\right)$. Minimum changes required to achieve $50 \%$ and $80 \%$ of the optimal effectiveness for both $L S T_{d}$ and $L S T_{n}$ are also marked.

\section{Discussions}

4.1 Variances in the LST-land-use relationship. The estimated LST-land-use relationships substantially vary spatially, diurnally and compositionally. Our findings in the context of EHEs agree with existing studies on regular hot days ${ }^{18,43}$ that substantial spatial variances exist in the LSTland-use relationships, especially for such high-impact land-use types as high-density urban lands. The responses of both daytime and nighttime LSTs can be profoundly affected by compositions of the urban lands, such as the development densities ${ }^{19,44}$, due to the so-called "canyon effect"15. The reduced air ventilation determined by local energy balance and stability prevents the heat from leaving the urban canopy layer trapping heat even after sunset.

However, this effect is not always true. For some areas in Shenzhen on the southeast coast, highdensity urban lands are found to contribute negatively to the nighttime LST. High-rise structures in densely built-up areas could reduce the amount of insolation from getting into the urban canopy. Also, the most important meteorological variable that alters the urban heat island effect is wind speed ${ }^{15}$. The identified areas in Shenzhen are located on the seaside, where the local thermal environment could benefit largely from the good air ventilation driven by sea and land breezes allowing urban areas to cool down swiftly after sunset. Such 'unexpected' phenomena further demonstrate the necessity of considering the varying effects, especially when dealing with spatial data and environmental phenomena such as urban warming.

4.2 Policy Implications of climate-conscious urban growth. Our results agree with Stone et al. ${ }^{4}$ that cities with a greater urban sprawl would be more vulnerable to EHEs. However, beyond that, our evidence suggests that not only the magnitude but also the spatial arrangement of urban growth matters to the associated warming impacts. We further summarize the dominant ( $\mathrm{P} .00 .5)$ change 
for each spatial location from the Pareto-optimal solutions and analyzed their structural (Figure 4) and spatial (Figure 5) patterns to provide suggestions for urban planners and researchers.

Structurally, climate-conscious urban growth in Shenzhen emphasizes compact urban development for minimal warming impacts (Figure 4). The CUGP introduces substantially more high-density (44.8\%) and low-density (35.7\%) urban lands than mid-density urban land (19.5\%).

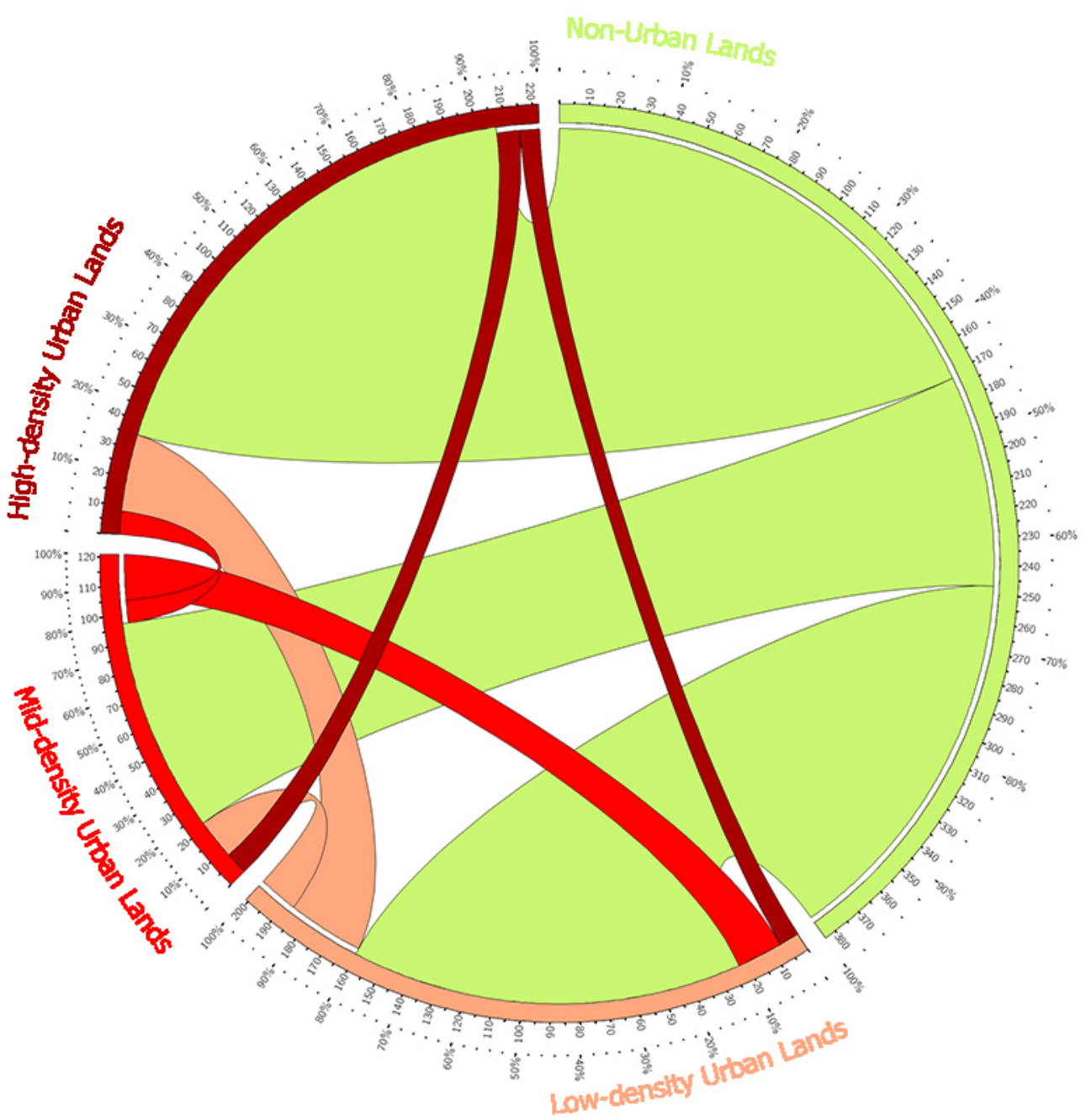

Figure 4 The structure of dominant land-use changes (P. $\geq 0.5)$ optimized by the CUGP, visualized as incoming and outgoing flows among the urban and non-urban land-use types using a circular plot ${ }^{43}$. Numbers on the inner circle indicate numbers of land parcels, while numbers on the outer circle are percentages of changed land parcels out of all land parcels in each land-use category.

Climate-conscious urban growth in Shenzhen also suggests a spatially equalized distribution of urban growth by locating more urban developments in the less-developed east half of Shenzhen (Figure 5). The centers of the optimized urban growth move eastward by an average of $19.1 \mathrm{~km}$ compared to the random urban growth (Figure S5). By optimizing the spatial positioning of urban lands, the CUGP also maximizes the mitigation effects of existing cooling sources in the city, 
including green space and water bodies. The percentages of cooling sources surrounding highdensity and mid-density urban lands are increased by the CUGP (Table S5, SI). Clusters of new urban lands (A, B, and C in Figure 5) are located adjacent to vast green spaces or water bodies. Such a spatial pattern suggests that "cooling sources" should be established and preserved even in compact cities. With sufficient "cooling sources", the CUGP could efficiently mitigate urban climate impacts of urban growth by locating compact urban development in places with sizeable biological capacity, ideal weather conditions, and room for urban growth.

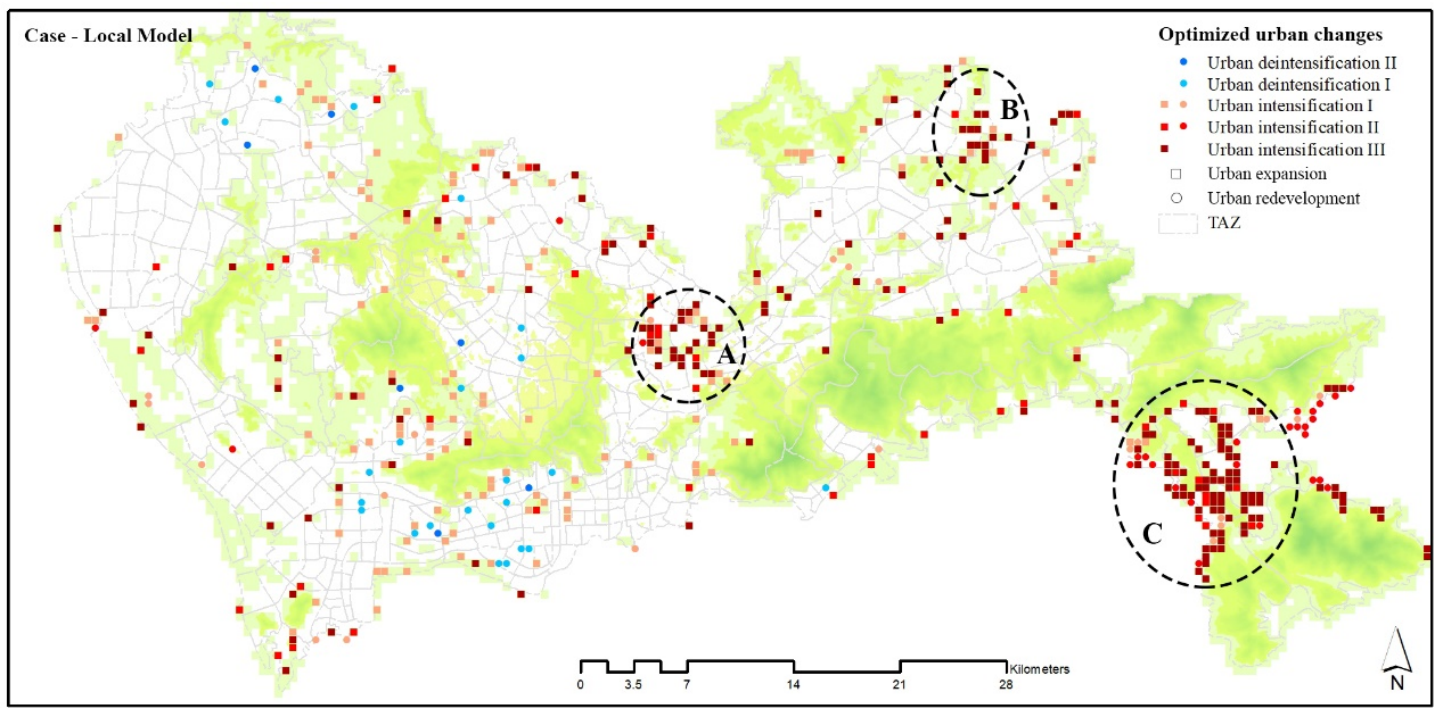

Figure 5. The spatial distribution of dominant urban changes (P. $\geq 0.5)$ optimized by the CUGP. The changes are classified as the urban expansion, i.e., urban development on newly acquired urban land, and urban redevelopment on existing urban land. Both categories are reclassified into several levels of urban intensification and urban deintensification to reflect the changes in urban densities (Table S4).

4.3 Model Sensitivity. We further examined the sensitivity of the mitigation effect using different combinations of model parameters. We repeated the analysis using three urban growth rates $(10 \%$, $20 \%$, and $30 \%$ ) and two elevation thresholds $(80 \mathrm{~m}$ and $120 \mathrm{~m})$. Results from the sensitivity analysis (Table S3) show that the mitigation effects were statistically significant $(p<0.005)$ using all the 12 parameter combinations. Please refer to Section S2.2.2 for more details.

\subsection{Future Perspectives and Limitations}

Unlike the simple and universal rules in the game of Go, real-world environmental problems are far more complex and include substantial spatio-temporal heterogeneities. Our results also show that artificial intelligence algorithms are highly sensitive to model specifications and need to work in a better-represented real-world settings.

For future directions, more evidence on the effectiveness of CUGP as an urban warming mitigation measure may still be needed, especially from cities of the developing world where massive urban growth is expected to occur, and local people are particularly vulnerable to extreme heat exposures. 
Moreover, since our objective is to investigate the optimal effectiveness of the CUGP, the effects of property rights, land markets, local land policies such as the Basic Ecological Control Line, and community benefits are not considered. Integrating the afore-mentioned factors in the investigation would provide more feasible land-use plans.

\section{Supporting Information}

- Supporting details about the experimental design, land-use map pre-processing, and the NSGA-II based SMOLA model.

- Detailed results from the LST-land-use relationship estimation, land-use optimization, and parameter sensitivity analysis.

\section{References}

(1) Howard, L. The Climate of London. Int. Assoc. Urban Clim. 1833, 285.

(2) Sharifi, E.; Lehmann, S. Comparative Analysis of Surface Urban Heat Island Effect in Central Sydney. J. Sustain. Dev. 2014, 7 (3), 23-34.

(3) Peng, S.; Piao, S.; Ciais, P.; Friedlingstein, P.; Ottle, C.; Bréon, F. M.; Nan, H.; Zhou, L.; Myneni, R. B. Surface Urban Heat Island across 419 Global Big Cities. Environ. Sci. Technol. 2012, 46 (2), 696-703.

(4) Stone, B.; Hess, J. J.; Frumkin, H. Urban Form and Extreme Heat Events: Are Sprawling Cities More Vulnerable to Climate Change than Compact Cities? Environ. Health Perspect. 2010, 118 (10), 1425-1428.

(5) Chen, Z.; Xie, X.; Cai, J.; Chen, D.; Gao, B.; He, B.; Cheng, N.; Xu, B. Understanding Meteorological Influences on PM2.5 Concentrations across China: A Temporal and Spatial Perspective. Atmos. Chem. Physic 2017, 0086 (0086), 1-30.

(6) Rizwan, A. M.; Dennis, L. Y. C.; Liu, C. A Review on the Generation, Determination and Mitigation of Urban Heat Island. J. Environ. Sci. 2008, 20 (1), 120-128.

(7) Santamouris, M. Using Cool Pavements as a Mitigation Strategy to Fight Urban Heat Island - A Review of the Actual Developments. Renewable and Sustainable Energy Reviews. 2013, pp 224 240.

(8) Santamouris, M. Cooling the Cities - A Review of Reflective and Green Roof Mitigation Technologies to Fight Heat Island and Improve Comfort in Urban Environments. Sol. Energy 2014, 103, 682-703.

(9) Kolokotsa, D.; Santamouris, M.; Zerefos, S. C. Green and Cool Roofs' Urban Heat Island Mitigation Potential in European Climates for Office Buildings under Free Floating Conditions. Sol. Energy 2013, 95, 118-130.

(10) Akbari, H.; Kolokotsa, D. Three Decades of Urban Heat Islands and Mitigation Technologies Research. Energy Build. 2016, 133, 834-852.

(11) Zhang, W.; Huang, B. Land Use Optimization for a Rapidly Urbanizing City with Regard to 
Local Climate Change: Shenzhen as a Case Study. J. Urban Plan. Dev. 2014, 141 (1), 5014007.

(12) Zhang, Y.; Murray, A. T.; Turner, B. L. Optimizing Green Space Locations to Reduce Daytime and Nighttime Urban Heat Island Effects in Phoenix, Arizona. Landsc. Urban Plan. 2017, 165, $162-171$.

(13) Heaviside, C.; Vardoulakis, S.; Cai, X. X.-M. Attribution of Mortality to the Urban Heat Island during Heatwaves in the West Midlands, UK. Environ. Heal. 2016, 15 (S1), S27.

(14) Urban Growth. In The New Palgrave Dictionary of Economics; Durlauf, S. N., Blume, L. E., Eds.; Nature Publishing Group: Basingstoke, 2008; pp 544-546.

(15) Oke, T. R. The Energetic Basis of the Urban Heat Island. Q. J. R. Meteorol. Soc. 1982, 108 (455), $1-24$.

(16) Buyantuyev, A.; Wu, J. Urban Heat Islands and Landscape Heterogeneity: Linking Spatiotemporal Variations in Surface Temperatures to Land-Cover and Socioeconomic Patterns. Landsc. Ecol. 2010, 25 (1), 17-33.

(17) Zhou, W.; Huang, G.; Cadenasso, M. L. Does Spatial Configuration Matter? Understanding the Effects of Land Cover Pattern on Land Surface Temperature in Urban Landscapes. Landsc. Urban Plan. 2011, 102 (1), 54-63.

(18) Zhou, X.; Wang, Y. C. Dynamics of Land Surface Temperature in Response to Land-Use/Cover Change. Geogr. Res. 2011, 49 (1), 23-36.

(19) Imhoff, M. L.; Zhang, P.; Wolfe, R. E.; Bounoua, L. Remote Sensing of the Urban Heat Island Effect across Biomes in the Continental USA. Remote Sens. Environ. 2010, 114 (3), 504-513.

(20) Wang, J.; Huang, B.; Fu, D.; Atkinson, P. M.; Zhang, X. Response of Urban Heat Island to Future Urban Expansion over the Beijing-Tianjin-Hebei Metropolitan Area. Appl. Geogr. 2016, 70, 2636.

(21) Tewari, M.; Salamanca, F.; Martilli, A.; Treinish, L.; Mahalov, A. Impacts of Projected Urban Expansion and Global Warming on Cooling Energy Demand over a Semiarid Region. Atmos. Sci. Lett. 2017, 18 (11), 419-426.

(22) Peng, J.; Xie, P.; Liu, Y.; Ma, J. Urban Thermal Environment Dynamics and Associated Landscape Pattern Factors: A Case Study in the Beijing Metropolitan Region. Remote Sens. Environ. 2016, 173, 145-155.

(23) Chapman, S.; Watson, J. E. M.; Salazar, A.; Thatcher, M.; McAlpine, C. A. The Impact of Urbanization and Climate Change on Urban Temperatures: A Systematic Review. Landsc. Ecol. 2017, 32 (10), 1921-1935.

(24) Connors, J. P.; Galletti, C. S.; Chow, W. T. L. L. Landscape Configuration and Urban Heat Island Effects: Assessing the Relationship between Landscape Characteristics and Land Surface Temperature in Phoenix, Arizona. Landsc. Ecol. 2013, 28 (2), 271-283.

(25) Zheng, B.; Myint, S. W.; Fan, C. Spatial Configuration of Anthropogenic Land Cover Impacts on Urban Warming. Landsc. Urban Plan. 2014, 130 (1), 104-111.

(26) Doan, V. Q.; Kusaka, H. Projections of Urban Climate in the 2050s in a Fast-Growing City in Southeast Asia: The Greater Ho Chi Minh City Metropolitan Area, Vietnam. Int. J. Climatol. 2018, 38 (11), 4155-4171. 
(27) Kusaka, H.; Suzuki-Parker, A.; Aoyagi, T.; Adachi, S. A.; Yamagata, Y. Assessment of RCM and Urban Scenarios Uncertainties in the Climate Projections for August in the 2050s in Tokyo. Clim. Change 2016, 137 (3-4), 427-438.

(28) Schlager, K. J. A Land Use Plan Design Model. J. Am. Plan. Assoc. 1965, 31 (2), 103-111.

(29) Cooper, L. Location-Allocation Problems. Oper. Res. 2008, 11 (3), 303-473.

(30) Wright, J. J.; Revelle, C.; Cohon, J.; Cohon Jared. A Multiobjective Integer Programming Model for the Land Acquisition Problem. Reg. Sci. Urban Econ. 1983, 13, 31-53.

(31) Stewart, T. J.; Janssen, R.; van Herwijnen, M. A Genetic Algorithm Approach to Multiobjective Land Use Planning. Comput. Oper. Res. 2004, 31 (14), 2293-2313.

(32) Ligmann-Zielinska, A.; Church, R.; Jankowski, P. Spatial Optimization as a Generative Technique for Sustainable Multiobjective Land-Use Allocation. Int. J. Geogr. Inf. Sci. 2008, 22 (6), 601-622.

(33) Janssen, R.; van Herwijnen, M.; Stewart, T. J.; Aerts, J. C. J. H. Multiobjective Decision Support for Land-Use Planning. Environ. Plan. B Plan. Des. 2008, 35 (4), 740-756.

(34) Cao, K.; Batty, M.; Huang, B.; Liu, Y.; Yu, L.; Chen, J. Spatial Multi-Objective Land Use Optimization: Extensions to the Non-Dominated Sorting Genetic Algorithm-II. Int. J. Geogr. Inf.

Sci. 2011, 25 (12), 1949-1969.

(36) Balling, R. J.; Taber, J. T.; Brown, M. R.; Day, K. Multiobjective Urban Planning Using Genetic

(37) Xiao, N.; Bennett, D. A.; Armstrong, M. P. Using Evolutionary Algorithms to Generate Alternatives for Multiobjective Site-Search Problems. Environ. Plan. A 2002, 34 (4), 639-656.

(39) Huang, B.; Zhang, W. Sustainable Land-Use Planning for a Downtown Lake Area in Central

(40) Luo, M.; Lau, N. C. Heat Waves in Southern China: Synoptic Behavior, Long-Term Change,

(41) Wan, Z. New Refinements and Validation of the Collection-6 MODIS Land-Surface

(42) Nakaya, T.; Fotheringham, S.; Charlton, M.; Brunsdon, C. Semiparametric Geographically

(43) Su, Y.-F.; Foody, G. M.; Cheng, K.-S. Spatial Non-Stationarity in the Relationships between 
A Preprint - Environmental Science \& Technology

Please Cite: http://dx.doi.org/10.1021/acs.est.9b01645

520

521

522

523
Cities: Spatial Patterns and Drivers. Remote Sens. Environ. 2014, 152, 51-61.

(45) Abel, G. J.; Sander, N. Quantifying Global International Migration Flows. Science (80-. ). 2014, 343 (6178), 1520-1522. 